\title{
A network pharmacology perspective for deciphering potential mechanisms of action of Solanum nigrum L. in bladder cancer
}

Yang Dong ${ }^{1,2+}$, Lin Hao ${ }^{1,2+}$, Kun Fang ${ }^{3 \dagger}$, Xiao-xiao Han ${ }^{4+}$, Hui Yu ${ }^{5}$, Jian-jun Zhang ${ }^{6}$, Long-jun Cai ${ }^{6}$, Tao Fan ${ }^{1}$, Wen-da Zhang ${ }^{1}$, Kun Pang ${ }^{1}$, Wei-ming Ma ${ }^{1}$, Xi-tao Wang ${ }^{1}$ and Cong-hui Han ${ }^{1,2,7^{*}}$

\begin{abstract}
Background: Solanum nigrum L. decoction has been used as a folklore medicine in China to prevent the postoperative recurrence of bladder cancer (BC). However, there are no previous pharmacological studies on the protective mechanisms of this activity of the plant. Thus, this study aimed to perform a systematic analysis and to predict the potential action mechanisms underlying S. nigrum activity in BC based on network pharmacology.

Methods: Based on network pharmacology, the active ingredients of S. nigrum and the corresponding targets were identified using the Traditional Chinese Medicines for Systems Pharmacology Database and Analysis Platform database, and BC-related genes were screened using GeneCards and the Online Mendelian Inheritance in Man database. In addition, ingredient-target (I-T) and protein-protein interaction (PPI) networks were constructed using STRING and Cytoscape, Gene Ontology (GO) terms and Kyoto Encyclopedia of Genes and Genomes (KEGG) pathway enrichment analyses were conducted, and then the pathways directly related to BC were integrated manually to reveal the pharmacological mechanism underlying S. nigrum-medicated therapeutic effects in BC.

Results: Seven active herbal ingredients from 39 components of S. nigrum were identified, which shared 77 common target genes related to BC. I-T network analysis revealed that quercetin was associated with all targets and that NCOA2 was targeted by four ingredients. Besides, interleukin 6 had the highest degree value in the PPI network, indicating a hub role. A subsequent gene enrichment analysis yielded 86 significant $\mathrm{GO}$ terms and 89 significant pathways, implying that S. nigrum had therapeutic benefits in BC through multi-pathway effects, including the HIF-1, TNF, P53, MAPK, PI3K/Akt, apoptosis and bladder cancer pathway.

(Continued on next page)
\end{abstract}

\footnotetext{
* Correspondence: 201706014207bjk@ncist.edu.cn

†Yang Dong, Lin Hao, Kun Fang and Xiao-xiao Han contributed equally to this work.

'Department of Urology, XuZhou Central Hospital Affiliated to Nanjing University of Chinese Medicine, Jiefang South Road, No. 199, Jiangsu,

Xuzhou, China

${ }^{2}$ Department of Clinical Medicine, Xuzhou Medical University, Xuzhou, China

Full list of author information is available at the end of the article
}

(c) The Author(s). 2021 Open Access This article is licensed under a Creative Commons Attribution 4.0 International License, which permits use, sharing, adaptation, distribution and reproduction in any medium or format, as long as you give appropriate credit to the original author(s) and the source, provide a link to the Creative Commons licence, and indicate if changes were made. The images or other third party material in this article are included in the article's Creative Commons licence, unless indicated otherwise in a credit line to the material. If material is not included in the article's Creative Commons licence and your intended use is not permitted by statutory regulation or exceeds the permitted use, you will need to obtain permission directly from the copyright holder. To view a copy of this licence, visit http://creativecommons.org/licenses/by/4.0/. The Creative Commons Public Domain Dedication waiver (http://creativecommons.org/publicdomain/zero/1.0/) applies to the data made available in this article, unless otherwise stated in a credit line to the data. 
(Continued from previous page)

Conclusions: S. nigrum may mediate pharmacological effects in BC through multi-target and various signaling pathways. Further validation is required experimentally. Network pharmacology approach provides a predicative novel strategy to reveal the holistic mechanism of action of herbs.

Keywords: Bladder cancer, Enrichment analysis, Network pharmacology, Solanum nigrum L., Target prediction

\section{Background}

Despite being one of the most common malignancies worldwide, bladder urothelial carcinoma remains refractory, and its incidence and mortality rates have been the highest among all genitourinary tumors for many years in China [1]. Although bladder cancer (BC) is mostly not invasive to muscles at the initial presentation, it regularly recurs, causing fatigue in most patients, and approximately $30 \%$ of patients eventually progress to muscle-invasive $\mathrm{BC}$ with 5 years [2]. In patients with localized, advanced-stage $\mathrm{BC}$, platinum-based combination chemotherapy continues to be the first line of therapy [3]. Despite the increased use of multimodality therapy with neoadjuvant chemotherapy followed by radical cystectomy or bladder sparing approaches [4-6], the longterm survival rates of patients with muscle-invasive $\mathrm{BC}$ have remained largely unchanged for decades $[7,8]$, and approximately $50 \%$ of cases progress to incurable metastatic BC [3, 9]. It has been reported that the use of immunotherapy with checkpoint inhibitors (CPIs) improves the outcomes of patients with metastatic disease $[3,8,10]$. In recent years, five CPIs, including three programmed cell death ligand-1 (PD-L1) inhibitors, namely atezolizumab, avelumab, and durvalumab, and two anti-PD-1 antibodies, nivolumab and pembrolizumab, have been approved by the Food and Drug Administration as systemic therapeutic agents for patients with metastatic and muscle-invasive $\mathrm{BCs}[3,10]$. However, the efficacy and safety of CPIs in non-muscle-invasive $\mathrm{BC}$ are currently under investigation in multiple clinical trials [10]. Although CPIs revolutionized the treatment of $\mathrm{BC}$, only approximately $20 \%$ of patients respond to immunotherapy, and the overall prognosis remains dismal [3].

Transurethral resection of bladder tumor followed by intravesical administration with Bacillus CalmetteGuérin or chemotherapy agents such as mitomycin, valrubicin, or gemcitabine is usually associated with a high incidence of local and systemic side effects, such as cystitis-like irritative voiding symptoms, hematuria, skin rash, arthralgia, fever, and influenza-like symptoms, which result in treatment discontinuation in $4-7 \%$ of patients [2]. Besides, adjuvant chemotherapy is generally accompanied by significant toxicity, primarily manifesting in the form of anemia, neutropenia, fatigue, nausea, and vomiting [9]. In addition to the toxicity of immune checkpoint inhibitors, the prohibitive costs of such drugs limit their clinical application $[2,10]$. Consequently, the development of novel and more effective and economical therapeutic strategies of BC treatment with low or even no toxicity or side effects is required.

Natural plants have been considered as an important source of therapeutic agents for human health for a long time. The beneficial effects of phytochemical drugs on the prevention and treatment of tumors have recently attracted much interest in the field of modern biomedicine. Traditional Chinese medicine (TCM) is composed of derivatives from natural plants, which has a long history of use as remedies for diseases with the focus lying on maintaining the dynamic balance of the entire body to achieve a state of harmony between the human being and nature. Solanum nigrum L., a herbal plant indigenous to Southeast Asia, is frequently used as a valuable ingredient in clinical TCM cancer therapy [11]. A previous study has shown that $S$. nigrum leaves contains a compound that can induce autophagy in breast adenocarcinoma cells [12] and apoptosis in hepatoma cells [13]. The mature fruits of $S$. nigrum can inhibit cell growth and, in turn, promote apoptosis in breast cancer cells [14]. By altering the expression of matrix metalloproteinases and inhibiting epithelial-mesenchymal transition, $S$. nigrum extracts effectively inhibits the invasion of prostate cancer cells [15]. In addition, a water extract of S. nigrum suppresses melanoma metastasis [16]. Such findings demonstrate that $S$. nigrum can induce antineoplastic activity as a chemopreventive anti-cancer agent. Since a long time, S. nigrum decoction has been used as a folklore medicine in China to prevent postoperative $\mathrm{BC}$ recurrence; however, its specific mechanism of action has remained unclear. Its active ingredients, cellular targets, and the molecular mechanisms of action in BC are yet to be elucidated.

Network pharmacology, a novel advanced analytical technology, is currently popular owing to its reliability and efficiency in pharmacological research [17]. The key principle of network pharmacology involves applying a network-based approach to construct a multilevel network (drug-gene-path-disease) to explore the therapeutic effects and mechanisms of action of drugs in complex therapies at organizational and molecular levels [18]. Such therapies have the characteristics of wholeness and systematic behavior, which are consistent with the principles of a holistic view and dialectical treatment 
associated with TCM [19]. Such a high-efficiency research strategy is emerging as the preferred approach for studies investigating the pharmacological mechanisms of TCM herbs and formulas. The therapeutic targets and pathways of such herbs and formulations can subsequently be verified in in vivo and in vitro experiments.

In the present study, we aimed to systematically analyze and explore the active components, specific targets, and the precise molecular mechanisms of S. nigrum in BC based on network pharmacology approach. The basic scheme of the pharmacology of the integrated extraction system is illustrated in Fig. 1. We performed a preliminary screening of the active ingredients of $S$. nigrum, and then identified the potential molecular targets of the ingredients and retained those responsible for the occurrence and progression of BC. Subsequently, the interactions among the potential targets were investigated, and Gene Ontology (GO) terms classification and pathway enrichment analysis were further performed. Ultimately, we visualized an integrated " $S$. nigrum pathway" to provide a systematic overview of the potential molecular mechanisms of action of $S$. nigrum activity in $\mathrm{BC}$. The results of the present study might be able to provide a novel approach for an in-depth understanding of the antineoplastic activity of this TCM.

\section{Methods}

\section{Screening the active ingredients of S. nigrum}

The herb's name, "Solanum nigrum L.," was checked in The Plant List (http://www.theplantlist.org/; version 1.1). The major components of $S$. nigrum were identified using the Traditional Chinese Medicines for Systems Pharmacology Database and Analysis Platform (TCMSP; https://tcmspw.com/tcmsp.php; updated on May 31, 2014), which is a well-known Chinese herbal medicine database providing correlations between diseases, drugs, and targets. To explore the potential primary active ingredients, we evaluated each candidate's druggability based on its corresponding oral bioavailability $(\mathrm{OB})$ and drug likeness (DL) values predicted in TCMSP database. $\mathrm{OB}$ refers to the degree and speed of drug absorption, when administered through the mouth, into the circulatory system [20]. DL refers to the similarity between a compound and a known drug in terms of physicochemical properties and structural factors, including solubility, permeability, and stability, which usually allows the pharmacokinetic properties and safety of a compound to be assessed [21]. Generally, higher OB and DL values indicate that the compound will be more valuable for clinical application. As recommended by TCMSP, compounds meeting both the criteria of $\mathrm{OB} \geq 30 \%$ and $\mathrm{DL} \geq 0.18$ are perceived to have relatively high affinity, intrinsic activity, and pharmacological properties and can be considered as candidate ingredients for further studies [20-22]. The data of which part of S. nigrum the ingredients extracted from was obtained using the Phytochemical Composition Database (http://www. organchem.csdb.cn/scdb/main/plant_introduce.asp).

\section{Molecular target prediction}

Owing to the diversity of $S$. nigrum constituents, the herb can target a variety of proteins; therefore, target prediction is a key step in exploring the molecular mechanism underlying the pharmacological properties of $S$. nigrum. In the present study, target prediction was performed using the TCMSP database, which integrates information from the DrugBank and Herb Ingredients' Targets (HIT) databases and the Systems Drug Targeting (SysDT) model [22]. DrugBank is a comprehensive knowledgebase that focuses on combining detailed drug data with drug action and target information [23]. Experimentally validated association between herbal compounds and their corresponding targets could be retrieved from the HIT database [24]. As for compounds without validated targets, the SysDT model was used to predict the potential drug-target interaction, which was based on random deep forest and support vector machine [25]. In addition, the UniProt Knowledgebase (UniProtKB; www.uniprot.org/; updated on July 23, 2020) [26] was used to unify the non-canonical description of the identified targets in the "Homo sapiens" category, and the normalized molecular targets along with their corresponding gene symbols were finally retrieved.

\section{Identification of the targets of BC}

The genes associated with $\mathrm{BC}$ were collected from two sources. One was the GeneCards database, which is recognized for its comprehensive information on all annotated human genes, proteins, and diseases [27]. This database is user-friendly, integrative, and reliable; it aggregates information from 125 different databases, such as the HUGO Gene Nomenclature Committee, National Center for Biotechnology Information, Ensembl, and UniProtKB. Using the GeneCards database (https://www.genecards.org/; version 5.0), information on the related targets was retrieved easily by performing keyword-based searches. The other information resource used was the Online Mendelian Inheritance in Man (OMIM) database (http://www.omim.org/; updated on May 4, 2018), which catalogs current diseases with genetic components and links them to the relevant genes in the human genome [28]. Here, we searched the GeneCards and OMIM databases to identify BC-related targets, with "bladder cancer" as the keyword.

\section{Ingredient-target (I-T) network construction}

Cytoscape is a software platform for complex network analysis and visualization [29]. In the present study, we displayed the interactions between ingredients and targets 


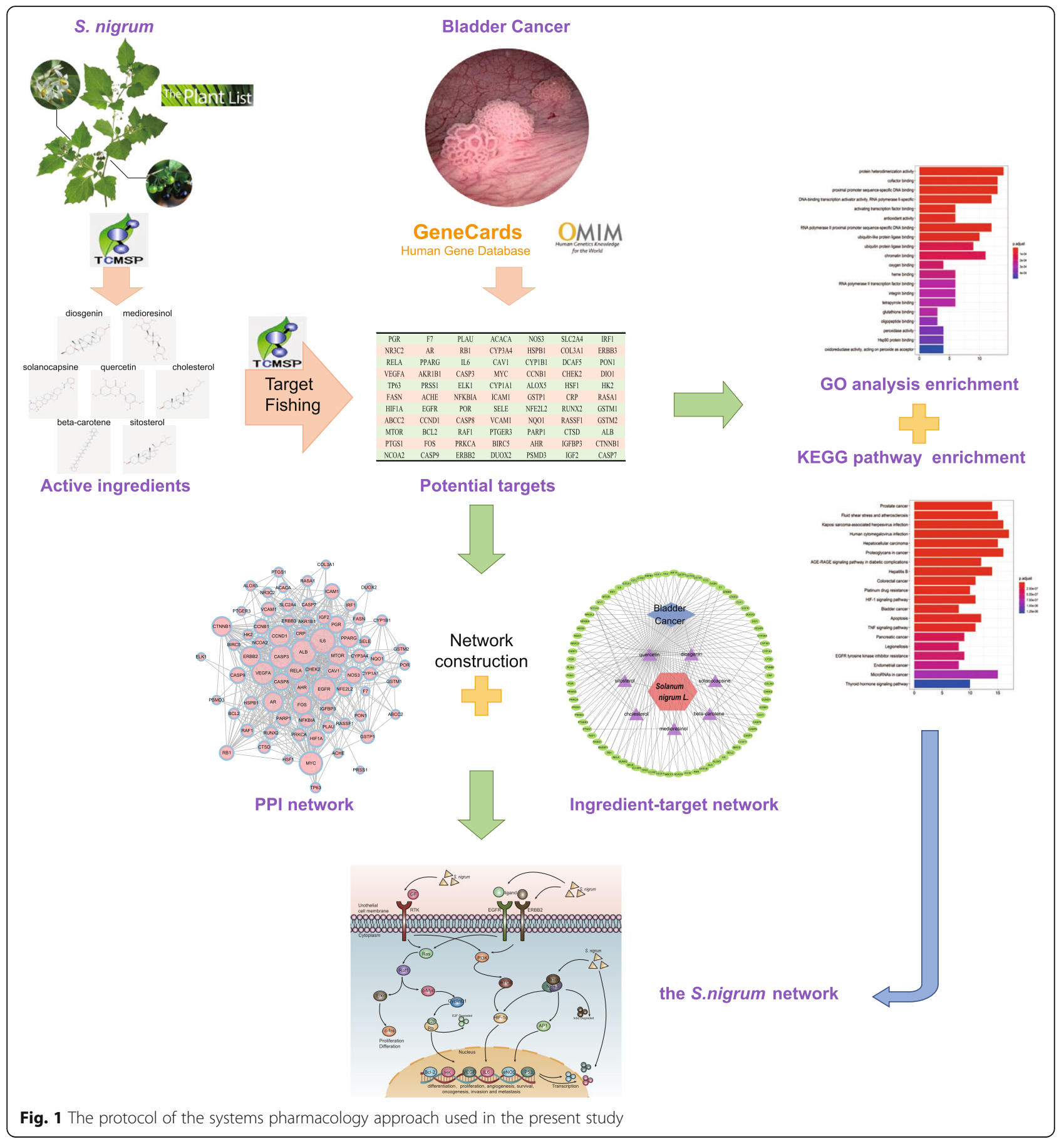

in a comprehensible manner, by constructing an I-T network using a graph visualization software, Cytoscape (version 3.7.1) (http://www.cytoscape.org/). The layout algorithm (attribute circle layout) was applied. To make the overall visual effect more distinct and easier to understand, Cytoscape allows users to adjust the geometric position of every node and symbol, the colors, and the graphics of the network topology depending on personal preferences. The degree and betweenness centrality are two of the most crucial parameters of the network's topological structure and are generally used to evaluate the essentiality of each part. These parameters facilitated the identification of the ingredients or targets that played key roles in the action of $S$. nigrum against BC.

\section{Protein-protein interaction network construction}

Internal proteins generally regulate a range of biological functions by interacting to form macromolecular 
complexes. Thus, exploring the interactions among proteins could provide a reliable theoretical foundation for clarifying the mechanisms of drug action, improving drug efficacy, and preventing adverse reactions. Search Tool for the Retrieval of Interacting Genes/Proteins (STRING) is one of the most extensively used graph visualization platforms for retrieving and predicting PPIs, with information obtained from experimental data, bioinformatics prediction, literature mining, and multiple databases [30]. To achieve a comprehensive understanding of the interactions among proteins, the relevant targets were inputted into STRING (https://string-db. org/; Version 11.0) for PPI network construction. Based on the intrinsic scoring mechanism, the higher the score, the more reliable the predictions of the interactions among proteins. We set the minimum value for the confidence at 0.4 and removed the disconnected proteins from the network. After importing the interaction data downloaded from STRING into the Cytoscape visualization software, the final PPI network was obtained and the number of edges per protein was tallied. In the network, nodes refer to proteins, whereas edges represent interactions among proteins.

\section{GO and pathway enrichment analyses}

The GO analysis can provide a standardized description and annotation for genes and gene products. Through GO analysis, users can obtain a comprehensive understanding from several aspects, including biological processes, cellular components, and molecular functions [31]. The Kyoto Encyclopedia of Genes and Genomes (KEGG) is a commonly used resource for the systematic analysis of gene functions and related high-level genome functional information [32, 33]. In the present study, GO and KEGG pathway enrichment analyses were conducted to explore the biological effects of $S$. nigrum using $\mathrm{R}$ the software (version 3.6) and three installed packages: (i) "DOSE," an R package for semantic similarity computations among disease ontology terms and genes, allowing biologists to explore the similarities of gene functions from the perspective of disease [34]; (ii) "clusterProfiler," an R package for comparing biological themes and enrichment analysis among gene clusters [35]; and (iii) "pathview," an R package for data visualization and integration based on the known pathways [36]. The corresponding $p$-value of each enriched term or pathway was calculated and corrected with Benjaminiand-Hochberg method to produce False Discovery Rate (FDR).

\section{Construction of the S. nigrum pathway}

To obtain an intuitive understanding of the mechanisms underlying the effect on S. nigrum in BC, an integrated "S. nigrum pathway" was manually developed based on the KEGG route map. Pathways not directly related to the disease were eliminated.

\section{Results \\ Identification of active ingredients}

In total, 39 ingredients of $S$. nigrum were retrieved through the TCMSP database query (Table A1). After identifying the ingredients that met the criteria of predicted $\mathrm{OB} \geq 30 \%$ and $\mathrm{DL} \geq 0.18$, seven active ingredients were finally retrieved: diosgenin (MOL000546), medioresinol (MOL002058), solanocapsine (MOL007356), quercetin (MOL000098), cholesterol (MOL000953), beta-carotene (MOL002773), and sitosterol (MOL000359) (as shown in Table 1). These active ingredients were considered to be mainly responsible for the therapeutic effects of $S$. nigrum.

\section{Target identification and analysis}

After target fishing using the TCMSP database, 84 potential targets were predicted to be regulated by the seven active ingredients of $S$. nigrum. Subsequently, by searching the GeneCards and OMIM databases for genes related to the occurrence and development of BC, 7624 genes were obtained. Integration of the targets regulated by $S$. nigrum and the targets related to $\mathrm{BC}$ revealed that 77 genes overlapped (shown in Table A2). Therefore, it is reasonable for us to consider that these genes are the potential therapeutic targets of S. nigrum in BC.

\section{I-T network construction and analysis}

To better understand the interaction between the candidate ingredients and the potential targets of S. nigrum in $\mathrm{BC}$, an I-T interaction network was constructed, in which each ingredient was linked to one or more target genes if the gene was a potential target of this ingredient. As shown in Fig. 2, the seven active ingredients were mapped to 77 potential target genes with 100 edges. The purple nodes represent the active ingredients and the green nodes represent the target genes. The edges indicate the interactions among them. Generally, the more genes an ingredient links to, the more likely it is to play a major role in the pharmacology of the herb. The degree of a node represents the number of routes connected to that node. The results of I-T network analysis showed that quercetin was connected to all targets with the highest degree value of 77 , which suggested that quercetin was more central than other active herbal ingredients, followed by beta-carotene (11 degrees) and diosgenin (9 degrees). In addition, 14 genes (myc protooncogene [MYC], HIF1 $\alpha, F 7, C Y B 3 A 4$, CAV1, RELA, caspase 3 [CASP3], CASP8, CASP9, BCL2, androgen receptor [AR], TP63, PTGS1, and peroxisome proliferator activated receptor gamma [PPARG]) had two degrees, three genes (progesterone receptor $[P G R]$, nuclear receptor subfamily 3 group $\mathrm{C}$ member 2 [NR3C2], and vascular endothelial growth factor A [VEGFA]) had three degrees, and one gene 
Table 1 The corresponding information and structures of the seven active components of Solanum nigrum with the predicted oral bioavailability $(\mathrm{OB}) \geq 30 \%$ and drug likeness $(\mathrm{DL}) \geq 0.18$

\begin{tabular}{|c|c|c|c|c|c|c|c|}
\hline No. & Molecule ID & Molecule name & MW & OB & $\mathrm{DL}$ & Parts & Structure \\
\hline 1 & MOL000546 & Diosgenin & 414.69 & 80.88 & 0.81 & $\begin{array}{l}\text { leaves } \\
\text { stems }\end{array}$ & \\
\hline 2 & MOL002058 & Medioresinol & 388.45 & 57.2 & 0.62 & fruits & \\
\hline 3 & MOL007356 & Solanocapsine & 430.75 & 52.94 & 0.67 & fruits & \\
\hline 4 & MOL000098 & Quercetin & 302.35 & 46.43 & 0.28 & $\begin{array}{l}\text { leaves } \\
\text { stems }\end{array}$ & \\
\hline 5 & MOL000953 & Cholesterol & 386.73 & 37.87 & 0.68 & fruits & \\
\hline 6 & MOL002773 & beta-Carotene & 536.96 & 37.18 & 0.58 & fruits & \\
\hline 7 & MOL000359 & Sitosterol & 414.79 & 36.91 & 0.75 & fruits & \\
\hline
\end{tabular}

(nuclear receptor coactivator 2 [NCOA2]) had four degrees, indicating that these genes were targeted by more than one active herbal ingredient.

\section{PPI network construction and analysis}

A PPI network of the targets of $S$. nigrum in the treatment of $\mathrm{BC}$ was constructed to analyze the interactions among proteins and to mine hub proteins.
After setting the confidence level to a value equal to or higher than 0.4 and after eliminating any protein not connected to the network, we obtained 77 proteins with 697 interrelations in the PPI network (Fig. 3a). A larger node size indicates a gene with a greater degree, which reflects a prioritization of the protein. Additionally, the gene with a prior degree value has been ranked in Fig. 3b. The degree value of 


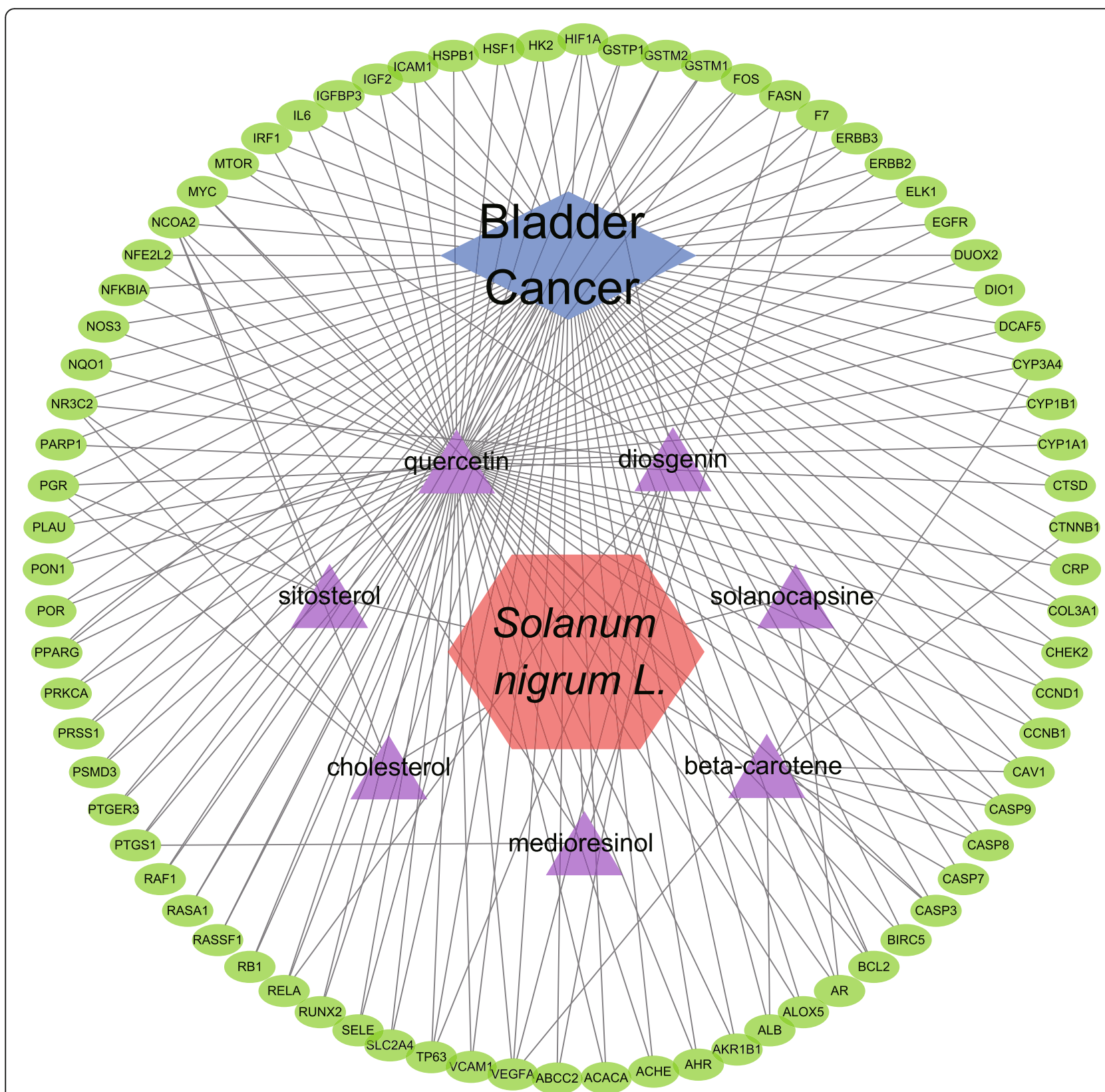

Fig. 2 The ingredient-target network generated in this study. The green nodes represent potential targets related to BC, and the purple nodes represent the active herbal ingredients; lines represent the interactions between them

interleukin 6 (IL-6) was 52, which was significantly higher than those of other proteins, indicating that IL-6 could act as a hub in the network. Besides, many proteins involved in tumor development in $\mathrm{BC}$ also showed high degree values, such as CASP3 and epidermal growth factor receptor (EGFR) (degrees of both are 47), MYC and VEGFA (degrees of both are 45), cyclin D1 (CCND1) (degree $=40)$, erb-b2 receptor tyrosine kinase $2($ ERBB2) (degree $=37)$, mechanistic target of rapamycin kinase $(\mathrm{mTOR}) \quad($ degree $=34)$, PPARG $($ degree $=33)$ and so on.

\section{GO functional enrichment analysis}

GO enrichment analysis was performed to elucidate the biological processes that the 77 genes participated in. Eighty-six remarkably enriched GO terms of these genes ( $p$-value $\leq 0.01$ ) were obtained. The 20 most significantly enriched GO terms are presented in Fig. 4a. The counts, $p$-values, and FDR of the significant terms are presented in Table A3. In summary, numerous genes were combined into one set, and many gene sets regulated specific biological activities involved in the occurrence and development of $\mathrm{BC}$, such as protein heterodimerization activity (GO: 0046982), 


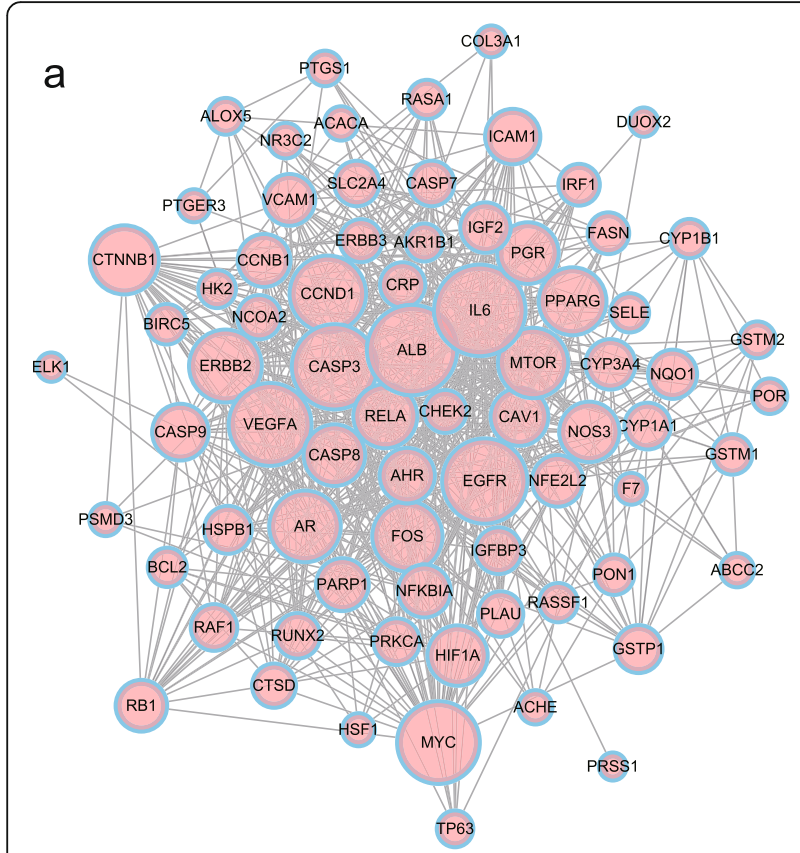

b

Fig. 3 a The protein-protein interaction (PPI) network of the potential targets associated with Solanum nigrum related to bladder cancer (BC). The nodes represent proteins, and the edges represent the interactions among them. The larger the node, the greater the degree of the node. b The bar plot of the first 30 target proteins sorted based on degree value. The longer the bar, the greater the connection of the protein

antioxidant activity (GO: 0016209), positive transcriptional regulation by RNA polymerase II promoter (GO: 0001085), nuclear receptor activity (GO: 0004879), and kinase regulator activity (GO: 0019207).

\section{KEGG pathway enrichment analysis}

The pathways related to the activity of S. nigrum in BC treatment were elucidated by KEGG pathway enrichment analysis. In total, 89 pathways were primarily enriched. The 20 most significantly enriched pathways ( $p$-value $\leq 0.01)$ are presented in Fig. $4 \mathrm{~b}$. The counts, $p$-values, and FDRs of the significant pathways are presented in Table A4. In summary, these genes were enriched in multiple pathways known to contribute to the tumorigenesis and progression of $\mathrm{BC}$, such as the HIF-1 signaling pathway (hsa04066), bladder cancer pathway (hsa05219), apoptosis

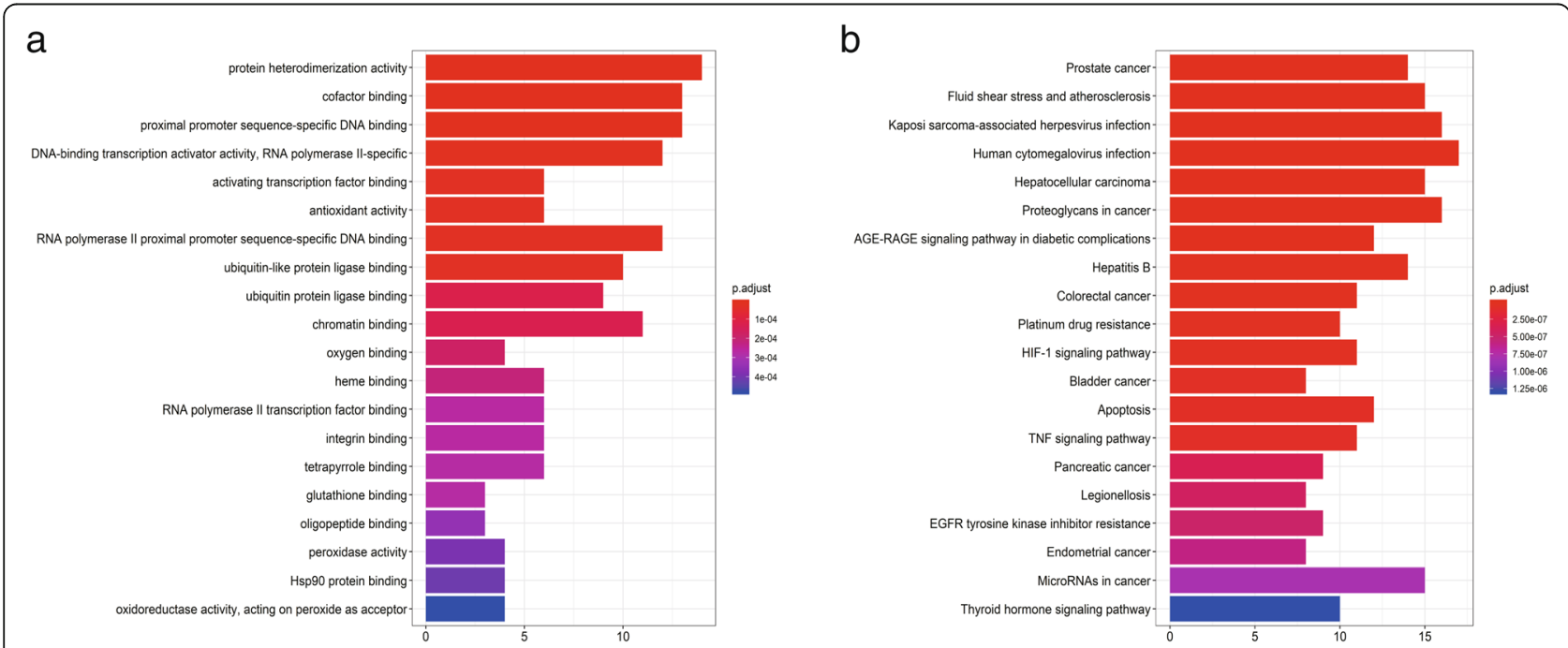

Fig. 4 a Gene ontology $(\mathrm{GO})$ analysis of the target genes associated with Solanum nigrum related to bladder cancer (BC); b KEGG pathway enrichment analysis of the target genes related to $B C$ that are modulated by $S$. nigrum. The $x$-axis represents significant enrichment counts of these terms, and the $y$-axis represents the categories of GO terms and KEGG pathways of the target genes ( $p$-value $\leq 0.01$ ) 
pathway (hsa04210), tumor necrosis factor (TNF) signaling pathway (hsa04668), p53 signaling pathway (hsa04115), mitogen-activated protein kinase (MAPK) signaling pathway (hsa04010), and PI3K/Akt signaling pathway (hsa04151).

\section{Integrated S. nigrum pathway construction}

To further comprehensively reveal the molecular mechanisms underlying the effects of $S$. nigrum against $\mathrm{BC}$, an integrated $S$. nigrum pathway model was assembled by combining the significant pathways associated with the occurrence and development of $\mathrm{BC}$ identified through
KEGG pathway enrichment analysis and some targets identified by I-T network analysis. Detailed information about the representative therapeutic modules to clarify the mechanisms of action of S. nigrum in BC was presented in Fig. 5. S. nigrum may exert anti-tumor effects in $\mathrm{BC}$ patients mainly by regulating the $\mathrm{BC}$ pathway (hsa05219), MAPK signaling pathway (hsa04010), HIF-1 signaling pathway (hsa04066), TNF signaling pathway (hsa04668), apoptosis signaling pathway (hsa04210), PI3K-Akt signaling pathway (hsa04151), p53 signaling pathway (hsa04115), and NF-kappa B signaling pathway (hsa04064).

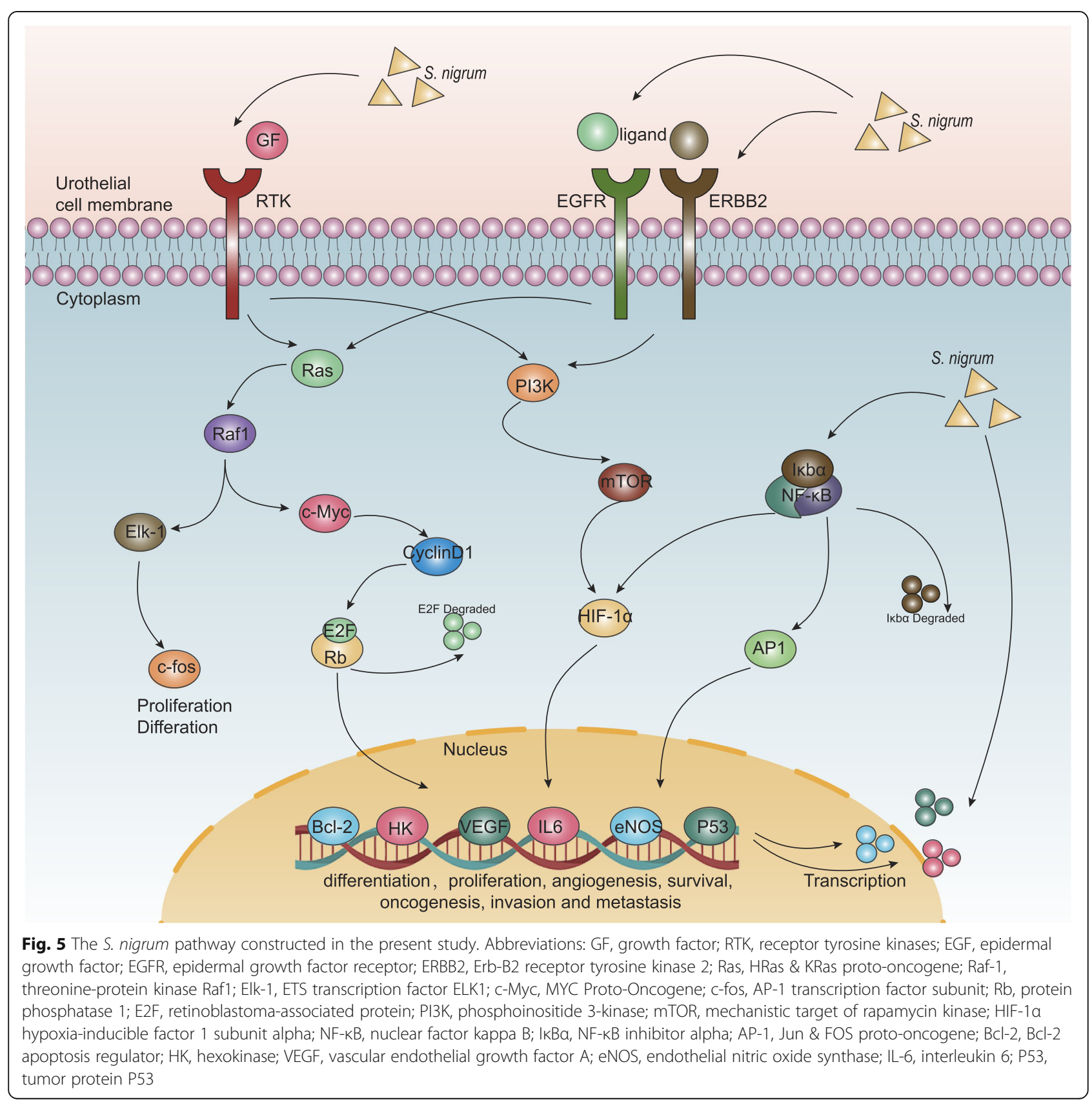




\section{Discussion}

$\mathrm{BC}$ is a heterogeneous disease [3] and the fifth most common malignancy globally [2]. Considering the characteristics of $\mathrm{BC}$, a key challenge in its treatment is the prevention of recurrence and progression to advanced stages [2, 8]. Although significant advances have been made in BC therapy with the advent of targeted therapies and immunotherapy, only a minority of patients benefit from the treatment and patient outcomes remain poor [3]. As a valuable alternative medicine, TCM has been associated with rich theoretical resources and invaluable experience that can be applied in clinical cancer therapy, and it has served as a vital resource for the development of multi-target drugs [19]. S. nigrum has been used to treat $\mathrm{BC}$ effectively [11]. Considering the component diversity of this herb, we used network pharmacology to comprehensively explore the mechanism of action of $S$. nigrum in BC treatment.

Via systems pharmacologic analysis, seven active ingredients were finally selected and 77 common target genes for $\mathrm{BC}$ were predicted. The seven identified bioactive compounds, including quercetin, sitosterol, diosgenin, medioresinol, beta-carotene, cholesterol and solanocapsine, are the major pharmacological constituents of S. nigrum. Quercetin, a flavonoid, belongs to an extensive class of polyphenolic compounds. It can not only inhibit the apoptosis and proliferation of numerous malignant cells, including $\mathrm{BC}$ and tumor growth in animal models through multiple signaling pathways [37], such as the AMPK [38], PI3K/Akt [39], and JNK pathways [40], but can also enhance the sensitivity of other anti-cancer agents and reverse the drug resistance of cancer cells [40]. Based on the I-T network, quercetin was linked to all targets with the highest degree among all selected ingredients, suggesting potential synergies between quercetin and all the other ingredients. Quercetin may act here as the main synergistic ingredient to increase the efficacy of $S$. nigrum as an anti-tumor agent. In addition, quercetin exhibits pharmacological activities as radical-scavenging antioxidant activity and antiinflammatory activity to inhibit carcinogenesis [41]. In clinical practice, a combination of cyclophosphamide and quercetin has been demonstrated to have good antineoplastic and antifatigue effects, as well as an excellent safety profile, in patients with BC [42].

Numerous studies have demonstrated that sitosterol exhibits potent anti-cancer activity against various malignancies, such as lung, breast, gastric, colon, and prostate cancers. Its potential mechanisms of action inducing apoptosis and inhibiting epithelial-mesenchymal transition through the blockade of multiple signaling pathways, including the MAPK, PI3K/Akt, Bcl-2, and Akt/ GSK-3 $\beta$ signaling pathways [43]. Diosgenin is a typical traditional medicine with multiple bioactivities, such as antidiabetic, anti-inflammatory, and anti-tumor properties [44]. Reportedly, most steroidal medicines are semisynthetic products obtained predominantly from diosgenin [45]. Medioresinol is a member of the lignan family, which are the predominant sources of phytoestrogens in western diets [46].

Dietary intake of plant lignans is beneficial for treating esophageal adenocarcinoma [47], colorectal cancer [48], and cardiovascular diseases and in the risk of hormonedependent cancers, such as breast, prostate, and endometrial cancers [46]. Beta-carotene mediates its therapeutic effects on human neuroblastoma, including the inhibition of cancer stemness and metastasis, by suppressing the enzyme activity and expression of MMPs, as well as the expression of HIF-1 $\alpha$ and its downstream targets [49]. Notably, unlike the other active ingredients, cholesterol in high content promotes inflammatory environments via the production of different cytokines, such as CXCL9 and CXCL10, but it also characterizes tumors, including breast, prostate, ovarian, lymphoma, colorectal cancer, and renal cell carcinoma, with poor prognosis [50]. Solanocapsine, a steroidal alkaloid, has shown potential therapeutic value as an inhibitor of acetylcholinesterase in patients with Alzheimer's disease [51]; however, its anticancer pharmacological effects are yet to be studied.

According to the I-T network, we observed that many genes were linked with at least two ingredients, indicating the synergistic properties of different ingredients of $S$. nigrum on BC. In our study, NCOA2, also commonly referred to as transcriptional intermediate factor 2, was predicted to be targeted by four active ingredients, including quercetin, sitosterol, medioresinol, and cholesterol. The activation of NCOA2 contributes to the progression of $\mathrm{BC}$, which is closely related to the activation of the EGFR and AR pathways [41]. In addition, the knockdown of $\mathrm{NCOA} 2$ results in a decrease in androgen-mediated cell proliferation in BC cell lines [42]. Preclinical evidence also reveals that AR inhibition can inhibit cancer cell growth and improve outcomes in patients with $\mathrm{BC}$ [3]. Therefore, NCOA2 is an attractive therapeutic target in $\mathrm{BC}$ treatment, which is largely consistent with our results. Moreover, three genes, $V E G F, P G R$, and NR3C2, are linked to three active ingredients, respectively. $V E G F$ is a well-known therapeutic target for many malignancies, including $\mathrm{BC}[52$, 53]. Increased PGR mRNA expression has been evaluated for its predictive value for non-muscle-invasive [54] and muscle-invasive $\mathrm{BC}$ [55], and this wellestablished $\mathrm{BC}$ marker is also a potential therapeutic target for $\mathrm{BC}$ [56]. NR3C2 has been described as a tumor suppressor in multiple malignancies, such as pancreatic, lung, colon, and renal cancers [57]; however, the prognostic value and biological effect of low NR3C2 level in BC remains unclear [58]. 
PPI network analysis revealed that the degree value of IL-6 was the highest, implying its role as a hub. The pro-inflammatory cytokine IL- 6 has been established as one of the most biologically active cytokines with multiple biological functions [19]. Numerous studies have demonstrated that the aberrant expression of IL-6 and its receptor correlates with malignant phenotypes of multiple tumors, as well as the diagnosis, prognosis, and treatment of cancer [59]. In one study, IL-6 was overexpressed in $\mathrm{BC}$ tissues compared with that in nonmalignant tissues, at both mRNA and protein levels, and was significantly associated with advanced clinical stage, high recurrence, and poor survival rate, indicating the diagnostic significance of IL-6 in BC [60]. Meanwhile, targeting IL- 6 might be a promising therapeutic target in BC because IL- 6 inhibition can attenuate bladder tumor growth and invasive capability [60]. S. nigrum can also reduce inflammation [61]. Therefore, targeting IL-6 is potentially one of the most effective pathways of $S$. nigrum-induced therapeutic effects on $\mathrm{BC}$.

In addition to IL-6, many proteins involved in the occurrence and development of $\mathrm{BC}$ also showed high degree values, such as CASP3, EGFR, MYC, VEGFA, CCND1, ERBB2, mTOR and PPARG. As known, CASP3 is a key enzyme in the execution of apoptosis and CCND1 codes for the cyclin D1 protein to affect the cell cycle progression [62], both of which were reported to contribute to many types of cancers and closely associated with cell motility, invasion, and metastasis [63]. EGFR overexpression is also found in a variety of human malignancies [64], including BC, which is implicated in stimulation of $\mathrm{BC}$ cell growth and induction of apoptosis [65] and appears to be a feasible target for BC therapy. ERBB2 also belongs to the EGFR family, which overexpression is associated with vascular growth and features of biological aggressiveness of BC [66]. MYC is known to function as an oncogene involved in the activation of various oncogenic signaling pathways, the inhibition of which can suppress cell growth and migration in $\mathrm{BC}$ [67] and cisplatin resistant BC cells [68]. PPARG could promote differentiation and regulate expression of mitochondrial genes in bladder epithelial cells, and its activation contributes to bladder carcinogenesis [69]. The importance of mTOR in carcinogenesis is becoming evident. In addition to relaying the oncogenic signals from the upstream PI3K/AKT pathway in various cancers, mTOR may play a direct role in human tumorigenesis if mutated, including in $\mathrm{BC}$, which further support the view of mTOR as one of the major therapeutic target against cancer [70]. These evidences all provide insights into the mechanistic function of $S$. nigrum as a bladder tumor suppressor.

Based on the GO classification and KEGG pathway enrichment results, $S$. nigrum interfered with the malignant phenotype of $\mathrm{BC}$ through diverse biological processes and signaling pathways. For example, the GO terms "RNA polymerase II," "protein heterodimerization activity," and "antioxidant activity" were closely associated with bladder carcinogenesis [71-73]. Among the enriched pathways, NF$\kappa B$ signaling pathway (hsa04064, degree $=7$ ) was confirmed to contribute to tumorigenicity and correlate with progression and prognosis in $\mathrm{BC}$ [74] and to participate in the metastasis-inhibiting effects of $S$. nigrum on melanoma cells [16]. The MAPK signaling pathway (hsa04010, degree $=14$ ), as one of the most intensively studied signaling pathways, has been found to be deregulated in various diseases, ranging from inflammatory and immunological syndromes to cancer, and controls the growth, proliferation, differentiation, and survival of various cells [75]. The results of an in vivo experiment suggest that targeting the MAPK pathway could be an effective therapy for BC [76]. In addition, S. nigrum could attenuate the malignant phenotype and tumor growth through the MAPK/mTOR signaling pathway in hepatocellular carcinoma [13] and the p38 signaling pathway in prostate cancer cells [77]. Therefore, MAPK signaling may be a crucial therapeutic target in the anti-tumor effects of S. nigrum. Furthermore, the upregulation of EGFR and ERBB2 protein levels was detected in numerous muscle-invasive $\mathrm{BC}$ samples, which induced PI3K (hsa04151, degree $=15$ ) and Ras activation, and were associated with $\mathrm{BC}$ grade, stage, and outcome [76]. As predicted in our study, EGFR and ERBB2 have been suggested to be targeted by quercetin [73]. Moreover, $S$. nigrum decreased blood serum TNF- $\alpha$ levels, which is consistent with the triggering of apoptosis in tumor cells [78]. Angiogenesis also plays a major role in different stages of tumor progression; therefore, some related pathways, such as the VEGF signaling pathway (hsa04370, degree $=6$ ) and the HIF-1 signaling pathway (hsa04066, degree $=11$ ), have been identified as the target pathways for treating $\mathrm{BC}[53$, 79]. All the results above are highly consistent with our findings from the GO and KEGG analyses. By integrating several previous well-established $\mathrm{BC}$-related pathways and the target genes identified by I-T network analysis, we assembled a proposed S. nigrum pathway model (Fig. 5). In theory $S$. nigrum might exert anti-BC effects mainly through acting on MAPK, HIF-1, TNF, PI3K-Ak, P53, NF-kappa B and apoptosis signaling pathways, which are importantly implicated in tumor cell growth, angiogenesis, invasion and metastasis, by directly regulating the expression of $E G F R, E E R B 2, G F, R A S$, RAF1, ELK1, FOS, MYC, CCND1, mTOR, HIF1,$N F \kappa B$, $B C L 2, H K, V E G F$ and $I L-6$. We hope that the pathway model facilitates a better understanding of the pharmacological mechanisms underlying the anti-cancer effects mediated by S. nigrum based on a comprehensive perspective.

S. nigrum has been reported to have dose-dependent toxic effects, resulting in nausea, vomiting, diarrhea, dizziness, headache, fever, sweating, tachycardia, loss of speech, blindness, mental confusion, coma, and death 
[80, 81]. A recent study reported that a patient suffered acute interstitial nephritis following the ingestion of $S$. nigrum and was successfully treated using corticosteroid therapy [81]. S. nigrum toxicity is mainly attributed to the presence of glycoalkaloids, including solanine, solasonine, and solamargine, which constitute the plant's primary natural defenses, as these compounds are toxic even at relatively low quantities [80]. S. nigrum has also been used to treat pain, inflammation, and fever [80]. Considering its widespread application for disparate ailments, more studies should be conducted to assess its toxicity and appropriate therapeutic dosage, as well as to explore standardized preparation methods, which would facilitate the optimization of therapeutic strategies, minimize side effects, and extend its potential benefits to more patients.

The present study had some limitations. First, the bioactive ingredients identified could differ from those actually absorbed by patients with BC. Secondly, it is difficult to distinguish the inhibitory target genes from the activated genes. In addition, not all predictions in the present study have been verified experimentally. Therefore, experimental verification of the active herbal ingredients is required to validate the hypotheses further.

\section{Conclusions}

The network pharmacology approach provides a predicative novel strategy for seeking evidence for the action mechanisms of herbal medicines based on a holistic perspective. Following target fishing and I-T network analysis, seven active ingredients acting on $77 \mathrm{BC}$-related genes were predicted, suggesting that $S$. nigrum might exert pharmacological effects against $\mathrm{BC}$ through multitargets. Quercetin linked to the large number of target genes may act as the main synergistic ingredient in $S$. nigrum, and NCOA2 was uniquely targeted by four ingredients. In addition, PPI network analysis indicated the hub role of IL-6. Additionally, gene enrichment analysis demonstrated that the active ingredients have potential therapeutic benefits with regard to $\mathrm{BC}$ via multiple pathways, such as the HIF-1, TNF, P53, MAPK, PI3K/Akt, apoptosis, and bladder cancer pathways. Nevertheless, further experiments are required to validate the theoretical predictions.

\section{Supplementary Information}

The online version contains supplementary material available at https://doi. org/10.1186/s12906-021-03215-3.

Additional file 1: Table A1. Thirty-nine components of Solanum nigrum and their corresponding molecular weight (MW), predicted oral bioavailability (OB) and drug likeness (DL) scores. Table A2. Bladder cancer (BC) related targets in Solanum nigrum. By combining the related targets of active ingredients screened from S. nigrum and the disease-related targets, 100 overlapping targets (involving 23 duplicates) were selected as the key targets involved in the treatment of BC. Table A3. Gene ontology $(\mathrm{GO})$ terms of therapeutic target genes and their corresponding counts, $p$-value, and FDR. Through the GO enrichment of the key targets, 86 remarkably enriched ( $p$-value $\leq 0.01$ ) GO terms were obtained, indicating that some targets were involved in tumorigenesis. Table A4. Kyoto Encyclopedia of Genes and Genomes (KEGG) pathway of therapeutic target genes and their corresponding counts, $p$-value, and FDR. Through KEGG enrichment of the key targets, 89 remarkably enriched ( $p$-value $\leq 0.01$ ) pathways were obtained, indicating that numerous targets are involved in the processes of tumorigenesis and tumor progression.

\section{Abbreviations}

BC: Bladder urothelial carcinoma; TCM: Traditional Chinese medicine; OB: Oral bioavailability; DL: Drug likeness; GO: Gene Ontology; KEGG: Kyoto

Encyclopedia of Genes and Genomes; OMIM: Online Mendelian Inheritance in Man; FDR: False Discovery Rate; MYC: Myc proto-oncogene;

CASP3: Caspase 3; AR: Androgen receptor; PGR: Progesterone receptor; PPARG: Peroxisome proliferator activated receptor gamma; NR3C2: Nuclear receptor subfamily 3 group C member 2; VEGFA: Vascular endothelial growth factor A; NCOA2: Nuclear receptor coactivator 2; IL-6: Interleukin 6; EGFR: Epidermal growth factor receptor; CCND1: Cyclin D1; ERBB2: Erb-b2 receptor tyrosine kinase 2; mTOR: Mechanistic target of rapamycin kinase

\section{Acknowledgments}

The authors acknowledge language editing support from Editage (www. editage.com).

\section{Authors' contributions}

$Y D, L H, X X H$, and $C H H$ conceived and designed the research. YD, XXH, LH and JJZ analyzed the data and wrote the manuscript. LJC and TF participated in target prediction and analysis. WDZ and KP performed the GO and KEGG enrichment analyses. YD, WMM, and XTW analyzed the data and optimized the graphs. $\mathrm{YD}, \mathrm{LH}$ and $\mathrm{CHH}$ revised the data and improved the manuscript. YD, LH, KF, HY and CHH participated in manuscript revision. All authors reviewed the manuscript, and all authors read and approved of the final version of the manuscript.

\section{Funding}

This work was supported by the Natural Science Foundation of China [grant numbers 81603498 and 81774089]; the Medical Innovation Team Project of Jiangsu Province [grant number CXTD-2016-48]; the Key Research and Development Project of Jiangsu Province [grant numbers BE2015623 and BE2017635]; the Young Medical Talent Project of Jiangsu Province [grant number QNRC2016386]; and the Traditional Chinese Medicine Bureau of Science and Technology Project of Jiangsu Province [grant number YB2017055]. The funding agencies had no role in study design; in the collection, analysis and interpretation of data; in the writing of the report; and in the decision to submit the article for publication.

\section{Availability of data and materials}

The datasets used and analyzed during the current study are available from the corresponding author on reasonable request.

Ethics approval and consent to participate

No humans or animals were used in this study; therefore, ethics approval and consent for participation were not required.

Consent for publication

All authors consent for publications.

\section{Competing interests}

The authors declare that they have no competing interests.

\section{Author details}

'Department of Urology, XuZhou Central Hospital Affiliated to Nanjing University of Chinese Medicine, Jiefang South Road, No. 199, Jiangsu, Xuzhou, China . ${ }^{2}$ Department of Clinical Medicine, Xuzhou Medical University, Xuzhou, China. ${ }^{3}$ Xuzhou Clinical Medical College of Integrated Traditional Chinese and Western Medicine Affiliated to Nanjing University of Traditional Chinese Medicine, Xuzhou, China. ${ }^{4}$ Center of Reproductive 
Medicine, Shanghai First Maternity and Infant Hospital, Tongji University School of Medicine, Shanghai, China. ${ }^{5}$ Yantai Hospital of Traditional Chinese Medicine, Yantai, China. ${ }^{6}$ Department of Urology, Suqian People's Hospital of Nanjing Drum-Tower Hospital Group, The Affiliated Suqian Hospital of Xuzhou Medical University, Suqian, China. ${ }^{7}$ Department of Biotechnology, College of Life Sciences, Jiangsu Normal University, Xuzhou, China.

\section{Received: 27 February 2020 Accepted: 11 January 2021}

\section{Published online: 25 January 2021}

\section{References}

1. Feng RM, Zong YN, Cao SM, Xu RH. Current cancer situation in China: good or bad news from the 2018 global Cancer statistics? Cancer Commun. 2019; 39(1):22.

2. Crijnen J, De Reijke TM. Emerging intravesical drugs for the treatment of non muscle-invasive bladder cancer. Expert Opin Emerg Drugs. 2018;23(2): 135-47.

3. Tripathi A, Gupta S. Androgen receptor in bladder cancer: a promising therapeutic target. Asian J Urol. 2020;7(3):284-90.

4. Vale C. Adjuvant chemotherapy in invasive bladder cancer: a systematic review and meta-analysis of individual patient data advanced bladder Cancer (ABC) meta-analysis collaboration. Eur Urol. 2005;48(2):189-99 discussion 199-201.

5. Vale C. Neoadjuvant chemotherapy in invasive bladder cancer: update of a systematic review and meta-analysis of individual patient data advanced bladder cancer (ABC) meta-analysis collaboration. Eur Urol. 2005;48(2):202-5 discussion 205-6.

6. Vashistha V, Quinn DI, Dorff TB, Daneshmand S. Current and recent clinical trials for perioperative systemic therapy for muscle invasive bladder cancer: a systematic review. BMC Cancer. 2014;14:966.

7. Zehnder P, Studer UE, Skinner EC, Thalmann GN, Miranda G, Roth B, et al. Unaltered oncological outcomes of radical cystectomy with extended lymphadenectomy over three decades. BJU Int. 2013;112(2):E51-8.

8. Gakis G. Management of muscle-invasive bladder cancer in the 2020s: challenges and perspectives. Eur Urol Focus. 2020;6(4):632-8.

9. Plimack ER, Hoffman-Censits JH, Viterbo R, Trabulsi EJ, Ross EA, Greenberg $R E$, et al. Accelerated methotrexate, vinblastine, doxorubicin, and cisplatin is safe, effective, and efficient neoadjuvant treatment for muscle-invasive bladder cancer: results of a multicenter phase II study with molecular correlates of response and toxicity. J Clin Oncol. 2014;32(18):1895-901.

10. Singh P, Black P. Emerging role of checkpoint inhibition in localized bladder cancer. Urol Oncol. 2016;34(12):548-55.

11. An L, Tang JT, Liu XM, Gao NN. Review about mechanisms of anti-cancer of Solanum nigrum. Zhongguo Zhong Yao Za Zhi. 2006;31(15):1225 -6, 1260

12. Huang HC, Syu KY, Lin JK. Chemical composition of Solanum nigrum Linn extract and induction of autophagy by leaf water extract and its major flavonoids in AU565 breast cancer cells. J Agric Food Chem. 2010;58(15): 8699-708.

13. Nath LR, Gorantla JN, Thulasidasan AK, Vijayakurup V, Shah S, Anwer S, et al. Evaluation of uttroside B, a saponin from Solanum nigrum Linn, as a promising chemotherapeutic agent against hepatocellular carcinoma [sci. rep.36318]. Sci Rep. 2016:6:36318.

14. Son YO, Kim J, Lim JC, Chung Y, Chung GH, Lee JC. Ripe fruit of Solanum nigrum L. inhibits cell growth and induces apoptosis in MCF-7 cells. Food Chem Toxicol. 2003:41(10):1421-8.

15. Shen KH, Liao AC, Hung JH, Lee WJ, Hu KC, Lin PT, et al. Alpha-solanine inhibits invasion of human prostate cancer cell by suppressing epithelialmesenchymal transition and MMPs expression. Molecules. 2014;19(8):11896914.

16. Wang HC, Wu DH, Chang YC, Li YJ, Wang CJ. Solanum nigrum Linn. Water extract inhibits metastasis in mouse melanoma cells in vitro and in vivo. J Agric Food Chem. 2010;58(22):11913-23.

17. Huang C, Zheng C, Li Y, Wang Y, Lu A, Yang L. Systems pharmacology in drug discovery and therapeutic insight for herbal medicines. Brief Bioinform. 2014;15(5):710-33.

18. Berger Sl, lyengar R. Network analyses in systems pharmacology. Bioinformatics. 2009;25(19):2466-72

19. Chen G, Huang C, Liu Y, Chen T, Huang R, Liang M, et al. A network pharmacology approach to uncover the potential mechanism of Yinchensini decoction. Evid Based Complement Alternat Med. 2018;2018: 2178610.
20. Xu X, Zhang W, Huang C, Li Y, Yu H, Wang Y, et al. A novel chemometric method for the prediction of human oral bioavailability. Int J Mol Sci. 2012; 13(6):6964-82.

21. Tao W, Xu X, Wang X, Li B, Wang Y, Li Y, Yang L. Network pharmacologybased prediction of the active ingredients and potential targets of Chinese herbal Radix Curcumae formula for application to cardiovascular disease. J Ethnopharmacol. 2013;145(1):1-10.

22. Ru J, Li P, Wang J, Zhou W, Li B, Huang C, et al. TCMSP: a database of systems pharmacology for drug discovery from herbal medicines. J Cheminform. 2014;6:13.

23. Wishart DS, Feunang YD, Guo AC, Lo EJ, Marcu A, Grant JR, et al. DrugBank 5.0: a major update to the DrugBank database for 2018. Nucleic Acids Res. 2018:46(D1):D1074-82

24. Ye H, Ye L, Kang H, Zhang D, Tao L, Tang K, et al. HIT: linking herbal active ingredients to targets. Nucleic Acids Res. 2011;39((database issue)):D1055-9.

25. Yu H, Chen J, Xu X, Li Y, Zhao H, Fang Y, et al. A systematic prediction of multiple drug-target interactions from chemical, genomic, and pharmacological data. PLoS One. 2012;7(5):e37608.

26. UniProt Consortium. UniProt: a worldwide hub of protein knowledge. Nucleic Acids Res. 2019;47(D1):D506-15.

27. Stelzer G, Rosen N, Plaschkes I, Zimmerman S, Twik M, Fishilevich S, et al. The GeneCards suite: from gene data mining to disease genome sequence analyses. Curr Protoc Bioinformatics. 2016;54(1):1.30.1-1.30.33.

28. Hamosh A, Scott AF, Amberger JS, Bocchini CA, McKusick VA. Online Mendelian Inheritance in Man (OMIM), a KnowledgeBase of human genes and genetic disorders. Nucleic Acids Res. 2005;33((database issue)):D514-7.

29. Shannon P, Markiel A, Ozier O, Baliga NS, Wang JT, Ramage D, et al. Cytoscape: a software environment for integrated models of biomolecular interaction networks. Genome Res. 2003;13(11):2498-504.

30. Szklarczyk D, Gable AL, Lyon D, Junge A, Wyder S, Huerta-Cepas J, et al. STR NG v11: protein-protein association networks with increased coverage, supporting functional discovery in genome-wide experimental datasets. Nucleic Acids Res. 2019;47(D1):D607-13.

31. The Gene Ontology Consortium. Expansion of the gene ontology KnowledgeBase and resources. Nucleic Acids Res. 2017;45(D1):D331-8.

32. Kanehisa $M$, Furumichi $M$, Tanabe $M$, Sato $Y$, Morishima K. KEGG: new perspectives on genomes, pathways, diseases and drugs. Nucleic Acids Res. 2017:45(D1):D353-61.

33. Kanehisa M, Goto S. KEGG: Kyoto encyclopedia of genes and genomes. Nucleic Acids Res. 2000;28(1):27-30.

34. Yu G, Wang LG, Yan GR, He QY. DOSE: an R/bioconductor package for disease ontology semantic and enrichment analysis. Bioinformatics. 2015; 31(4):608-9.

35. Yu G, Wang LG, Han Y, He QY. clusterProfiler: an R package for comparing biological themes among gene clusters. Omics. 2012;16(5):284-7.

36. Luo W, Brouwer C. Pathview: an R/bioconductor package for pathwaybased data integration and visualization. Bioinformatics. 2013;29(14):1830-1.

37. Ma L, Feugang JM, Konarski P, Wang J, Lu J, Fu S, et al. Growth inhibitory effects of quercetin on bladder cancer cell. Front Biosci. 2006;11:2275-85.

38. Su Q, Peng M, Zhang Y, Xu W, Darko KO, Tao T, et al. Quercetin induces bladder cancer cells apoptosis by activation of AMPK signaling pathway. Am J Cancer Res. 2016;6(2):498-508.

39. Chen F, Chen X, Yang D, Che X, Wang J, Li X, et al. Isoquercitrin inhibits bladder cancer progression in vivo and in vitro by regulating the PI3K/Akt and PKC signaling pathways. Oncol Rep. 2016;36(1):165-72.

40. Turner KA, Manouchehri JM, Kalafatis M. Sensitization of recombinant human tumor necrosis factor-related apoptosis-inducing ligand-resistant malignant melanomas by quercetin. Melanoma Res. 2018;28(4):277-85.

41. Yang CS, Landau JM, Huang MT, Newmark HL. Inhibition of carcinogenesis by dietary polyphenolic compounds. Annu Rev Nutr. 2001;21:381-406.

42. Di Lorenzo G, Pagliuca M, Perillo T, Zarrella A, Verde A, De Placido S, Buonerba C. Complete response and fatigue improvement with the combined use of cyclophosphamide and quercetin in a patient with metastatic bladder cancer: a case report. Medicine (Baltimore). 2016;95(5): e2598.

43. Cao ZQ, Wang XX, Lu L, Xu JW, Li XB, Zhang GR, et al. Beta-sitosterol and gemcitabine exhibit synergistic anti-pancreatic cancer activity by modulating apoptosis and inhibiting epithelial-mesenchymal transition by deactivating Akt/GSK-3beta signaling. Front Pharmacol. 2018;9:1525.

44. Yu H, Liu Y, Niu C, Cheng Y. Diosgenin increased DDX3 expression in hepatocellular carcinoma. Am J Transl Res. 2018;10(11):3590-9. 
45. Dong J, Lei C, Lu D, Wang Y. Direct biotransformation of dioscin into diosgenin in rhizome of Dioscorea zingiberensis by Penicillium dioscin. Indian J Microbiol. 2015;55(2):200-6.

46. Meija L, Söderholm P, Samaletdin A, Ignace G, Siksna I, Joffe R, et al. Dietary intake and major sources of plant lignans in Latvian men and women. Int J Food Sci Nutr. 2013;64(5):535-43.

47. Lin Y, Wolk A, Håkansson N, Lagergren J, Lu Y. Dietary intake of lignans and risk of esophageal and gastric adenocarcinoma: a cohort study in Sweden. Cancer Epidemiol Biomark Prev. 2013;22(2):308-12.

48. Cotterchio M, Boucher BA, Manno M, Gallinger S, Okey A, Harper P. Dietary phytoestrogen intake is associated with reduced colorectal cancer risk. J Nutr. 2006;136(12):3046-53.

49. Kim YS, Gong X, Rubin LP, Choi SW, Kim Y. Beta-carotene 15,15'-oxygenase inhibits cancer cell stemness and metastasis by regulating differentiationrelated miRNAs in human neuroblastoma. J Nutr Biochem. 2019;69:31-43.

50. Casaburi I, Chimento A, De Luca A, Nocito M, Sculco S, Avena P, et al. Cholesterol as an endogenous ERRalpha agonist: a new perspective to cancer treatment. Front Endocrinol. 2018;9:525.

51. García ME, Borioni JL, Cavallaro V, Puiatti M, Pierini AB, Murray AP, Peñéñory $A B$. Solanocapsine derivatives as potential inhibitors of acetylcholinesterase: synthesis, molecular docking and biological studies. Steroids. 2015;104:95110

52. van Kessel KE, Zuiverloon TC, Alberts AR, Boormans JL, Zwarthoff EC. Targeted therapies in bladder cancer: an overview of in vivo research. Nat Rev Urol. 2015;12(12):681-94.

53. Mazzola CR, Chin J. Targeting the VEGF pathway in metastatic bladder cancer. Expert Opin Investig Drugs. 2015;24(7):913-27.

54. Breyer J, Wirtz RM, Laible M, Schlombs K, Erben P, Kriegmair MC, et al. ESR1, ERBB2, and Ki67 mRNA expression predicts stage and grade of non-muscleinvasive bladder carcinoma (NMIBC). Virchows Arch. 2016;469(5):547-52.

55. Kriegmair MC, Wirtz RM, Worst TS, Breyer J, Ritter M, Keck B, et al. Prognostic value of molecular breast cancer subtypes based on Her2, ESR1, PGR and Ki67 mRNA-expression in muscle invasive bladder cancer. Transl Oncol. 2018;11(2):467-76.

56. Breyer J, Wirtz RM, Otto W, Laible M, Schlombs K, Erben P, et al. Predictive value of molecular subtyping in NMIBC by RT-qPCR of ERBB2, ESR1, PGR and MKI67 from formalin fixed TUR biopsies. Oncotarget. 2017:8(40):67684-95.

57. Zhao Z, Zhang M, Duan X, Deng T, Qiu H, Zeng G. Low NR3C2 levels correlate with aggressive features and poor prognosis in non-distant metastatic clearcell renal cell carcinoma. J Cell Physiol. 2018;233(10):6825-38.

58. Long MD, Campbell MJ. Pan-cancer analyses of the nuclear receptor superfamily. Nucl Recept Res. 2015;2:101182.

59. Vainer N, Dehlendorff C, Johansen JS. Systematic literature review of IL-6 as a biomarker or treatment target in patients with gastric, bile duct, pancreatic and colorectal cancer. Oncotarget. 2018;9(51):29820-41.

60. Chen MF, Lin PY, Wu CF, Chen WC, Wu CT. IL-6 expression regulates tumorigenicity and correlates with prognosis in bladder cancer. PLoS One. 2013;8(4):e61901.

61. Zhao L, Wang L, Di SN, Xu Q, Ren QC, Chen SZ, et al. Steroidal alkaloid solanine a from Solanum nigrum Linn. Exhibits anti-inflammatory activity in lipopolysaccharide/interferon gamma-activated murine macrophages and animal models of inflammation. Biomed Pharmacother. 2018;105:606-15.

62. Clemente-Vicario F, Alvarez CE, Rowell JL, Roy S, London CA, Kisseberth WC, et al. Human genetic relevance and potent antitumor activity of heat shock protein 90 inhibition in canine lung adenocarcinoma cell lines. PLoS One. 2015;10(11):e0142007.

63. Hu Y, Gu Y, Wang H, Huang Y, Zou YM. Integrated network model provides new insights into castration-resistant prostate cancer. Sci Rep. 2015;5:17280.

64. Shigeishi H, Sugiyama M, Tahara H, Ono S, Kumar Bhawal U, Okura M, et al. Increased telomerase activity and hTERT expression in human salivary gland carcinomas. Oncol Lett. 2011;2(5):845-50.

65. Chang L, Shi R, Yang T, Li F, Li G, Guo Y, et al. Restoration of LRIG1 suppresses bladder cancer cell growth by directly targeting EGFR activity. J Exp Clin Cancer Res. 2013;32:101.

66. Soria F, Moschini M, Haitel A, Wirth GJ, Gust KM, Briganti A, et al. The effect of HER2 status on oncological outcomes of patients with invasive bladder cancer. Urol Oncol. 2016;34(12):533 e531-10

67. Daga M, Pizzimenti S, Dianzani C, Cucci MA, Cavalli R, Grattarola M, et al. Ailanthone inhibits cell growth and migration of cisplatin resistant bladde cancer cells through down-regulation of Nrf2, YAP, and c-Myc expression. Phytomedicine. 2019;56:156-64.
68. Sun J, Zhang H, Tao D, Xie F, Liu F, Gu C, et al. CircCDYL inhibits the expression of C-MYC to suppress cell growth and migration in bladder cancer. Artif Cells Nanomed Biotechnol. 2019;47(1):1349-56.

69. Liu C, Tate T, Batourina E, Truschel ST, Potter S, Adam M, et al. Pparg promotes differentiation and regulates mitochondrial gene expression in bladder epithelial cells. Nat Commun. 2019;10(1):4589.

70. Pinto-Leite R, Arantes-Rodrigues R, Sousa N, Oliveira PA, Santos L. mTOR inhibitors in urinary bladder cancer. Tumour Biol. 2016;37(9):11541-51.

71. Riese DJ, Cullum RL. Epiregulin: roles in normal physiology and cancer. Semin Cell Dev Biol. 2014;28:49-56.

72. Ceci C, Lacal PM, Tentori L, De Martino M, Miano R, Graziani G. Experimental evidence of the antitumor, antimetastatic and antiangiogenic activity of ellagic acid. Nutrients. 2018;10(11):1756

73. Young RA. RNA polymerase II. Annu Rev Biochem. 1991;60:689-715.

74. Mukherjee N, Houston TJ, Cardenas E, Ghosh R. To be an ally or an adversary in bladder cancer: the NF-KB story has not unfolded. Carcinogenesis. 2015;36(3):299-306.

75. Orton RJ, Sturm OE, Vyshemirsky V, Calder M, Gilbert DR, Kolch W. Computational modelling of the receptor-tyrosine-kinase-activated MAPK pathway. Biochem J. 2005;392(2):249-61.

76. Knowles MA, Hurst CD. Molecular biology of bladder cancer: new insights into pathogenesis and clinical diversity. Nat Rev Cancer. 2015:15(1):25-41.

77. Pan B, Zhong W, Deng Z, Lai C, Chu J, Jiao G, et al. Inhibition of prostate cancer growth by solanine requires the suppression of cell cycle proteins and the activation of ROS/P38 signaling pathway. Cancer Med. 2016;5(11): 3214-22.

78. Li J, Li Q, Feng T, Zhang T, Li K, Zhao R, et al. Antitumor activity of crude polysaccharides isolated from Solanum nigrum Linne on U14 cervical carcinoma bearing mice. Phytother Res. 2007;21(9):832-40.

79. Streeter $\mathrm{EH}$, Harris AL. Angiogenesis in bladder cancer - prognostic marker and target for future therapy. Surg Oncol. 2002;11(1-2):85-100.

80. Jain R, Sharma A, Gupta S, Sarethy IP, Gabrani R. Solanum nigrum: current perspectives on therapeutic properties. Altern Med Rev. 2011:16(1):78-85.

81. Oh SS, Choi MW, Choi MR, Lee JH, Yang HJ, Choi YJ, et al. Acute interstitial nephritis induced by Solanum nigrum. Kidney Res Clin Pract. 2016;35(4):252-4.

\section{Publisher's Note}

Springer Nature remains neutral with regard to jurisdictional claims in published maps and institutional affiliations.

Ready to submit your research? Choose BMC and benefit from:

- fast, convenient online submission

- thorough peer review by experienced researchers in your field

- rapid publication on acceptance

- support for research data, including large and complex data types

- gold Open Access which fosters wider collaboration and increased citations

- maximum visibility for your research: over $100 \mathrm{M}$ website views per year

At BMC, research is always in progress.

Learn more biomedcentral.com/submissions 